\title{
Effect of Incudostapedial Reconstruction Using Conchal Cartilage Interposition Graft on Hearing Outcome
}

\author{
Nazrin Hameed ${ }^{1} \quad$ Arun Alexander $^{1}{ }^{\circledR} \quad$ Sunil Kumar Saxena ${ }^{1} \quad$ Sivaraman Ganesan $^{1}$ Jyotirmay S. Hegde ${ }^{1}$ \\ ${ }^{1}$ Ear, Nose and Throat Department, Jawaharlal Institute of \\ Postgraduate Medical Education \& Research, Puducherry, India \\ Int Arch Otorhinolaryngol 2019;23:e262-e266.

\begin{abstract}
Address for correspondence Arun Alexander, MS, DNB, Ear, Nose and Throat Department, Jawaharlal Institute of Postgraduate Medical Education \& Research, Old Hospital Block, Dhanvantri Nagar, Gorimedu, Jipmer Campus, Puducherry, Tamil Nadu, 605006, India
\end{abstract} \\ (e-mail: arunalexandercmc@gmail.com).
}

\begin{abstract}
Introduction Chronic otitis media is a widely prevalent condition in developing countries and is a cause of correctable hearing loss. The most frequent ossicular chain defect found during surgery for chronic otitis media has been a discontinuity of the incudostapedial joint. This study observes the effect of incudostapedial reconstruction using conchal cartilage on the hearing of the patient.

Objectives To evaluate improvement in hearing by incudostapedial reconstruction using conchal cartilage interposition graft in tympanoplasty and to identify the independent factors associated with erosion of the long process of incus among patients with chronic suppurative otitis media tubotympanic type.

Methods This study was conducted in the department of ear, nose and throat (ENT) amongst 22 patients with tubotympanic-type chronic suppurative otitis media who were found to have erosion of the long process during tympanoplasty. These patients underwent incudostapedial reconstruction using conchal cartilage. Their hearing was again reassessed at 12 weeks postsurgery, and the changes were observed.

Results A statistically significant improvement in air conduction by $15.14 \mathrm{~dB}$ was found after undergoing incudostapedial reconstruction using conchal cartilage $(p<0.001)$. There was no statistically significant change in bone conduction ( $p$ value $>0.05$ ). A total of $59.1 \%$ of patients in the study had an improvement in hearing

Keywords

- tympanoplasty

- cartilage

- graft

- incus ranging from 11 to $20 \mathrm{~dB}$. It was also found that $50 \%$ of the patients had a postoperative hearing of 10 to $20 \mathrm{~dB}$.

Conclusion Conchal cartilage interposition graft effectively improved hearing when used for the reconstruction of the incudostapedial joint during tympanoplasty in patients of chronic suppurative otitis media.
\end{abstract}

\section{Introduction}

Chronic suppurative otitis media (CSOM) is characterized by chronic inflammation of the mucosa of the middle ear cleft, leading to purulent otorrhea. Chronic suppurative otitis media (1D) Arun Alexander's ORCID is https://orcid.org/0000-0003-1026-
4678 .

received

February 26, 2018

accepted

May 8, 2018
DOI https://doi.org/

10.1055/s-0038-1661398. ISSN $1809-9777$. is a common condition prevalent in developing countries, such as India, and is more common in the lower socioeconomic groups. Many factors contribute to its widespread prevalence, including poor socioeconomic status, malnutrition, lack of hygiene and health awareness and immunocompromised status. It is equally prevalent among males and females of all age groups. Chronic suppurative otitis media is a leading cause of correctable hearing loss among the rural population.

Copyright @ 2019 by Thieme Revinter

Publicações Ltda, Rio de Janeiro, Brazil

License terms

c) $(1) \$$ 
Ossicular erosion is a sequela that is commonly associated with chronic otitis media. The most frequent ossicular chain defect found during surgery for chronic otitis media has been a discontinuity of the incudostapedial joint. ${ }^{1-3}$ Even though resorption can occur at any part of the ossicles, the most commonly involved parts are the long process of the incus and the capitulum and crura of stapes. The delicate structure as well as the tenuous blood supply to the lenticular process of the incus make the incudostapedial joint particularly vulnerable to resorption. ${ }^{4}$ The ossicular erosion in chronic otitis media has been attributed to be the result of hypervascularization, activation of osteoclast and resorption of bone caused by the inflammatory process, which leads to overproduction of cytokines, such as tumor necrosis factor $\alpha$, interleukin 2, fibroblast growth factor and platelet derived growth factor. ${ }^{5}$ The long process of incus and stapes superstructure, which are the finely constructed parts of the ossicular chain, are the most common parts to undergo necrosis due to the abundant osteoclastic activity but weak osteoblastic activity. The longer the duration of this inflammatory process and the closer it is to the ossicles, the higher the chances that it results in ossicular necrosis. ${ }^{6}$

The term tympanoplasty was introduced by Wullstein to describe the surgical techniques for the reconstruction of the middle ear hearing mechanism that had been impaired or destroyed by chronic ear disease. ${ }^{7}$ The methods of reconstruction vary from natural methods like ossicular repositioning, autograft materials like cortical bone and cartilage and homograft ossicles to artificial materials like plastic prosthesis, which was soon discarded because of its high extrusion rates, alloplastic ossicular prosthesis, partial ossicular replacement prosthesis, total ossicular replacement prosthesis, ceramic implants, hydroxyapatite and bone cement. ${ }^{8}$ Conchal cartilage has been used worldwide by otologic surgeons for more than 30 years for bridging ossicular chain defects, tympanic membrane reconstruction and repair of bony meatal wall defects. ${ }^{9-13}$

Chaudhary et al performed a study in 82 patients with tubotympanic-type CSOM who underwent ossiculoplasty using autogenous cartilage and autogenous bone for the repair of ossicular defect. As a result of this study, it was found that both autogenous cartilage and bone are effective in providing good hearing results postoperatively with $84 \%$ of the patients having a closure of air bone gap within $20 \mathrm{~dB}$, and $37 \%$ of patients having a closure of air bone gap within 10 $\mathrm{dB} .^{14}$ Autologous cartilage struts are found to be more suitable than homologous cartilage struts, and the preservation of perichondrium on at least one side increased the viability of the cartilage. ${ }^{15}$ Various studies about the role of autografts in the reconstruction of ossicular chain in intact canal wall procedures concluded that in the era when a large variety of innovative artificial prosthetic materials are used to replace and reconstruct the ossicular chain, autografts still play a significant role. Patients with CSOM had fairly good hearing results when implanted with autogenous cartilage. These are easily available and cost effective. Moreover, they are stable and are easily accepted by the body and never extruded out. ${ }^{14}$
In this study, conducted among patients with tubotympanic-type CSOM, we have analyzed the results of reconstruction of the defect between the eroded long process of incus and stapes head using conchal cartilage by comparing the results of the pure tone audiometry (PTA) tests taken preoperatively and 12 weeks postoperatively.

\section{Objectives}

\section{Primary Objective}

To evaluate the improvement in hearing achieved by incudostapedial reconstruction using conchal cartilage interposition graft in tympanoplasty.

\section{Secondary Objective}

To identify the independent factors, like age, gender, socioeconomic status and duration of disease, associated with erosion of the long process of incus.

\section{Methodology}

This is a 'before and after intervention study' conducted in the department of otorhinolaryngology in our institute from November 2015 to April 2017 among adult patients with tubotympanic-type chronic otitis media with a dry perforation undergoing tympanoplasty.

These patients were taken up for tympanoplasty and, during surgery, those patients who were found to have incudostapedial discontinuity were chosen for the study with the exclusion of those with erosion of more than half of the long process of incus. Convenient sampling technique was used and a total of 22 patients meeting the above-mentioned inclusion and exclusion criteria during the study period were chosen for this study. When the long process of incus was found to be eroded, a small rectangular piece of cartilage was excised from the posterior aspect of the concha using sharp dissection. The conchal cartilage was refashioned according to the size of the defect and was used to bridge the gap between the eroded long process and stapes head. The middle ear was packed with Gelfoam (Pfizer Inc., New York, NY, USA) and temporalis fascia graft was used to repair the tympanic membrane perforation in all cases (-Figs. 1, 2)

The independent variables included were age, gender, socioeconomic status and duration of disease, which was recorded in the data collection performa. Outcome variable was the average hearing loss in decibels for air conduction and bone conduction at 250,500, 1,000, 2,000 \& 4,000 Hz, which was recorded using PTA both preoperatively and 12 weeks postoperatively. The preoperative average hearing loss for air conduction and bone conduction was compared with the postoperative values recorded at 12 weeks.

Results on continuous measurements are presented as mean \pm standard deviation (SD) (min-max) and the results on categorical measurements are presented in numbers (\%). The mean and SD of hearing loss in $\mathrm{dB}$ pre and postoperatively were compared using the paired $t$-test. All statistical analyses were performed at $5 \%$ level of significance and a $p$ value $<0.05$ was considered significant. The statistical 


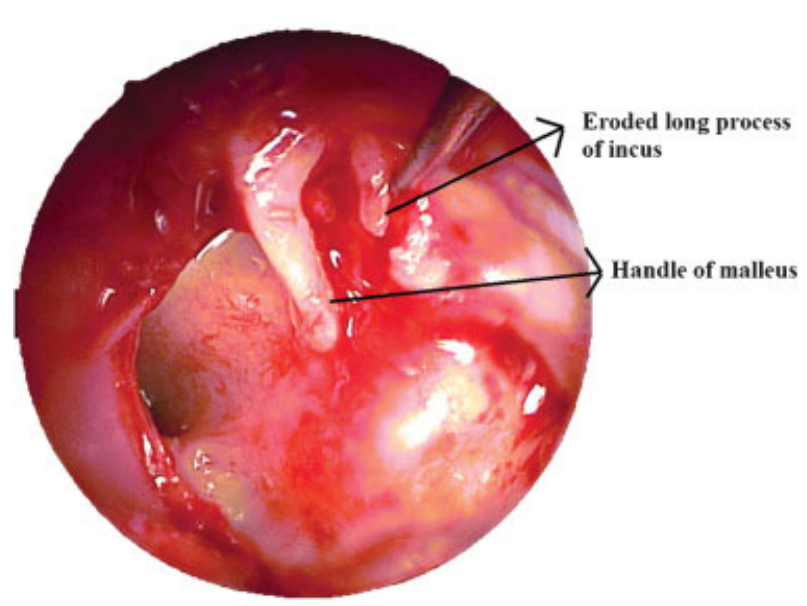

Fig. 1 Intraoperative picture showing erosion of the long process of incus.

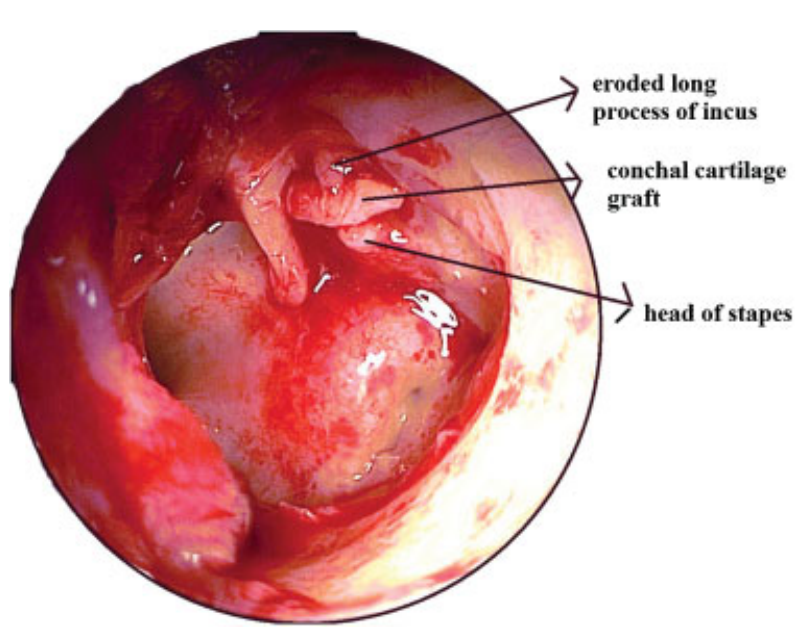

Fig. 2 Intraoperative picture showing conchal cartilage graft in situ.

software packages SAS 9.2 (SAS Institute, Cary, NC, USA) and SPSS 15.0 (SPSS Inc., Chicago, IL, USA) were used for the analysis of the data.

\section{Result}

Among the 22 patients who participated in this study, $68.2 \%$ were females. The mean age of the subjects with incus erosion was found to be 34.4 years, with a standard deviation of 10.95 , and most of the patients (36.4\%) were found to be between 31 to 40 years (-Tables 1, 2).

The socioeconomic status of the patients in the study was assessed using a modified Kuppuswamy scale, and it was found that $86.4 \%$ of patients were from lower socioeconomic status.

Table 1 Gender distribution of the patients studied

\begin{tabular}{|l|l|l|}
\hline Gender & No. of patients & $\%$ \\
\hline Male & 7 & 31.8 \\
\hline Female & 15 & 68.2 \\
\hline Total & 22 & 100.0 \\
\hline
\end{tabular}

Table 2 Age distribution of the patients studied

\begin{tabular}{|l|l|l|}
\hline Age in years & No. of patients & $\%$ \\
\hline$<20$ & 3 & 13.6 \\
\hline $20-30$ & 5 & 22.7 \\
\hline $31-40$ & 8 & 36.4 \\
\hline $41-50$ & 5 & 22.7 \\
\hline$>50$ & 1 & 4.6 \\
\hline Total & 22 & 100.0 \\
\hline
\end{tabular}

Mean \pm SD: $34.36 \pm 10.95$

The mean duration of symptoms was 8.5 years, with a standard deviation of 8.4 . However, the majority of the patients (45\%) had symptoms ranging from 1 to 5 years (-Tables 3,4 ).

The mean preoperative hearing loss for air conduction was found to be $40 \mathrm{~dB}$, with a standard deviation of 10.8 . It was also found that patients in the older age group ( $>35$ years) with incus erosion had more hearing loss as compared with the younger age group, and this difference was found to be statistically significant with a $p$ value of 0.047 (-Table 5).

The mean postoperative hearing loss for air conduction was found to be $24.9 \mathrm{~dB}$, with a standard deviation of 10.03 Thus, it was found from this study that there is a significant improvement in air conduction by $15.14 \mathrm{~dB}$ after undergoing incudostapedial reconstruction using conchal cartilage, and this difference has been found to be statistically significant, with a $p$ value $<0.001$. In contrast to the results of air conduction, it was noted that there was no statistically significant change in bone conduction, with a $p$ value $>0.05$ (-Table 6 ).

A total of $59.1 \%$ of the patients in the study had an improvement in hearing ranging from 11 to $20 \mathrm{~dB}$. It was also found that $50 \%$ of the patients had a postoperative hearing of 10 to $20 \mathrm{~dB}$ (-Figs. 3, 4).

Table 3 Socioeconomic status distribution of the patients studied

\begin{tabular}{|l|l|l|}
\hline Socioeconomic status & No. of patients & $\%$ \\
\hline Lower & 19 & 86.4 \\
\hline Lower middle & 1 & 4.5 \\
\hline Upper middle & 2 & 9.1 \\
\hline Total & 22 & 100.0 \\
\hline
\end{tabular}

Table 4 Duration of disease distribution of the patients studied

\begin{tabular}{|l|l|l|}
\hline Duration of disease (years) & No. of patients & $\%$ \\
\hline$<1$ & 5 & 22.7 \\
\hline$>1-<5$ & 10 & 45.5 \\
\hline$>5-<10$ & 4 & 18.2 \\
\hline$>10-<20$ & 4 & 18.2 \\
\hline$>20$ & 2 & 9.1 \\
\hline Total & 22 & 100.0 \\
\hline
\end{tabular}

Mean \pm SD: $8.48 \pm 8.40$ 
Table 5 Hearing loss $(\mathrm{dB})$ in relation to age (in years) distribution of patients studied

\begin{tabular}{|l|l|l|l|l|}
\hline \multirow{2}{*}{ Air conduction } & \multicolumn{2}{|l|}{ Age in years } & Total & \multirow{2}{*}{-value } \\
\cline { 2 - 3 } & $<35$ yrs & $>35$ years. & & \\
\hline Preop & $35.10 \pm 10.04$ & $44.17 \pm 10.02$ & $40.05 \pm 10.82$ & $0.047^{*}$ \\
\hline Postop & $20.80 \pm 11.65$ & $28.33 \pm 7.28$ & $24.91 \pm 10.03$ & $0.079+$ \\
\hline Difference & $14.30 \pm 6.57$ & $15.83 \pm 5.47$ & $15.14 \pm 5.90$ & 0.557 \\
\hline
\end{tabular}

Table 6 Comparison of Hearing $(\mathrm{dB})$ at pre and post op assessment

\begin{tabular}{|l|l|l|l|l|l|}
\hline Hearing & Preop & Postop & Difference & $t$ value $^{*}$ & $p$-value \\
\hline Air conduction $(\mathrm{dB})$ & $40.05 \pm 10.82$ & $24.91 \pm 10.03$ & 15.136 & 12.037 & $<0.001^{* *}$ \\
\hline Bone conduction $(\mathrm{dB})$ & $8.77 \pm 5.27$ & $8.14 \pm 4.40$ & 0.636 & 0.856 & 0.401 \\
\hline
\end{tabular}

${ }^{*}$ Student $t$-test (paired).

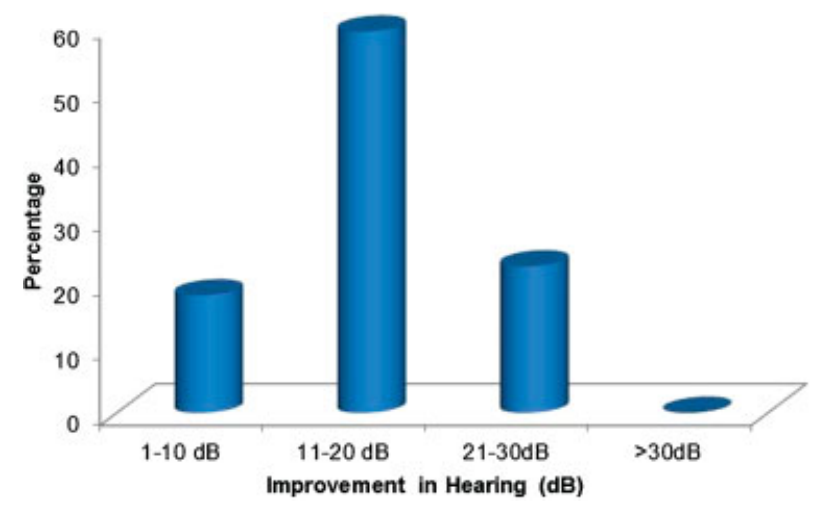

Fig. 3 Improvement in hearing of patients (dB)

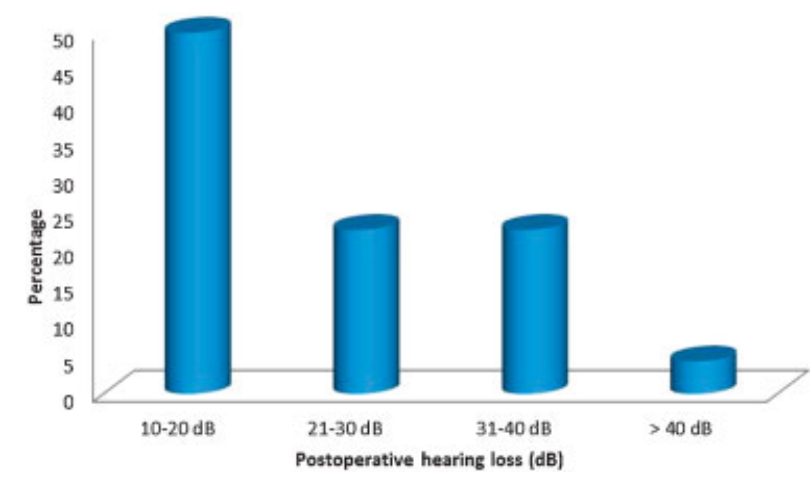

Fig. 4 Postoperative hearing of patients (dB).

\section{Discussion}

The mean preoperative hearing loss for air conduction was found to be $40 \mathrm{~dB}$, with a standard deviation of 10.8. This was similar to the results of a study by Mohanty et al, conducted amongst 20 patients in which necrosis of incus was associated with a mean hearing loss of $49.18 \mathrm{~dB}$, and Ebenezer et al, who predicted that incus erosion is associated with a hearing loss ranging from 40 to $70 \mathrm{~dB} .{ }^{16,17}$ This suggests that pure tone audiogram (PTA) is a reasonably good indicator of ossicular discontinuity and hence, options for appropriate reconstruc- tion methods can be considered preoperatively if PTA is suggestive of ossicular erosion. It was also found that patients in the older age group ( $>35$ years) with incus erosion had more hearing loss as compared with the younger age group, and this difference was found to be statistically significant with a $p$ value of 0.047 .

The mean postoperative hearing loss for air conduction was found to be $24.9 \mathrm{~dB}$, with a standard deviation of 10.03 . Thus, it was found from this study that there is a significant improvement in air conduction by $15.4 \mathrm{~dB}$ after undergoing incudostapedial reconstruction using conchal cartilage, and this difference has been found to be statistically significant with a $p$ value $<0.001$. This was consistent with the findings of a study by Galy-Bernadoy et al, in which they compared the hearing outcomes of type II tympanoplasty done with various biomaterials for erosion of long process of incus. ${ }^{18}$ In contrast to the results of air conduction, it was noted that there was no statistically significant change in bone conduction with $p$ value $>0.05$.

Another purpose of this study was to identify the independent factors, like age, gender, socioeconomic status and duration of disease, associated with erosion of the long process of incus. This study was conducted in a tertiary care hospital. Most of the patients visiting the hospital were referred from peripheral hospitals for surgical intervention. Among the 22 patients amongst whom this study was conducted, $68.2 \%$ were females thus showing female preponderance in tubotympanictype CSOM patients with incus erosion. This was, however, in contrast with the findings of Jayakumar et al, in whose study there was a high male preponderance for incus erosion (62.5\%), even though this study was conducted amongst tubotympanic-type CSOM patients amongst whom the vast majority were females $(68.1 \%)^{19}$

The mean age of the subjects with incus erosion was found to be 34.4years, with a standard deviation of 10.95 , and most of the patients (36.4\%) were found to be between 31 and 40 years. This was similar to the findings of Jayakumar et al, who conducted a study to assess preoperative indicators of ossicular necrosis in tubotympanic-type CSOM disease. ${ }^{19}$

The socioeconomic status of the patients in the study was assessed using a modified Kuppuswamy scale, and $86.4 \%$ of 
patients were from lower socioeconomic status. Low socioeconomic status has been found to be associated with the epidemiology of CSOM.

The main presenting symptom of the patients in our study was ear discharge followed by difficulty of hearing, and the mean duration of symptoms was 8.5 years, with a standard deviation of 8.4. However, the majority of the patients (45\%) had symptoms ranging from 1 to 5 years. This was, however, different from the findings of Jayakumar et al, who found that most patients with incus erosion had symptoms for more than 10 years. ${ }^{19}$ This change could be an indicator of increasing health awareness resulting in patients presenting early for health care.

Our study also showed that the majority of the patients (59.1\%) had an improvement of air conduction by 11 to $20 \mathrm{~dB}$, which is the same as that noted during the literature review. The final postoperative hearing achieved was in the range of 10 to $20 \mathrm{~dB}$ in $50 \%$ of the patients. This suggests that conchal cartilage interposition graft is indeed an effective method for the improvement of hearing in CSOM patients with erosion of the long process of incus, and it is comparable in efficacy to several other biomaterials that are available for the same purpose.

The advantage of this method is that conchal cartilage, being an autograft, is an easily available, safe and inexpensive material for the reconstruction of the incudostapedial joint. It can be harvested from the same field of surgery, thus not requiring any separate incisions and not adding to postoperative pain. It does not have any deleterious effects that are seen to be associated with artificial prosthetic materials like glass ionomer cement, which was found to be associated with serious complications like encephalopathy due to the presence of aluminum in its composition. It is also free of cost, unlike other artificial biomaterials such as glass ionomer cement and titanium prosthesis, which are expensive and thus have limited use in patients of low socioeconomic status, especially when CSOM has been found to be closely linked to poor socioeconomic status.

There have been many studies that have postulated that the long-term behavior of cartilage grafts is non-satisfactory, as cartilage grafts remained viable for only a certain length of time and would eventually show changes in their character and function, thus making them unfit as sound conductors. Our study, however, has evaluated postoperative hearing only at 12 weeks, and we are largely unaware of the plight of hearing as far as long-term benefits are concerned. If the study had been conducted over a longer time period, with regular follow-up of hearing loss, it would have offered more information regarding the long-term effect of conchal cartilage graft on hearing.

\section{Conclusion}

This before and after intervention study conducted among CSOM patients with incus erosion has showed that conchal cartilage interposition graft effectively improved hearing when used for the reconstruction of the incudostapedial joint during tympanoplasty in patients of tubotympanictype CSOM. The majority of the patients had a postoperative hearing within 10 to $20 \mathrm{~dB}$, which is comparable, if not better, to that achieved with several other biomaterials.

\section{Conflicts of Interest}

The authors have no conflicts of interest.

\section{Ethical Approval}

All procedures involving human participants were in accordance with the ethical standards of the institutional and/or national research committee, and with the 1964 Declaration of Helsinki and its amendments or comparable ethical standards. Ethical clearance was obtained from the Institute Ethics Committee, JIPMER (Reg. No. JIP/IEC/2015/20/714), and was registered in the Clinical Trials Registry of India (CTRI). Informed consent was obtained from all individual participants included in the study.

\section{References}

1 Feghali JG, Barrs DM, Beatty CW, et al. Bone cement reconstruction of the ossicular chain: a preliminary report. Laryngoscope 1998;108(06):829-836

2 Hafiz G. A more reliable method for incudostapedial rebridging ossiculoplasty: bone cement and wire. Adv Ther 2005;22(01):56-62

3 Maassen MM, Zenner HP. Tympanoplasty type II with ionomeric cement and titanium-gold-angle prostheses. Am J Otol 1998;19 (06):693-699

4 Glasscock ME, Shambaugh GE, Johnson GD. Surgery of the ear. 4. ed. Philadelphia, Pa London: W.B. Saunders; 1990

5 Anglitoiu A, Balica N, Lupescu S, Vintila R, Cotulbea S. Ossicular chain status in the otological pathology of the ENT clinic Timisoara. Med Evol 2011;17(04):344-351

6 Sade J, Berco E, Buyanover D, Brown M. Ossicular damage in chronic middle ear inflammation. Acta Otolaryngol 1981;92(34):273-283

7 Wullstein HL. Tympanoplasty: the fundamentals of the concept. Clin Otolaryngol Allied Sci 1978;3(04):431-435

8 Kartush JM, Balough BJ. Contemporary ossiculoplastic options. Curr Opin Otolaryngol Head Neck Surg 2001;9(05):272-278

9 Brockman SJ. Cartilage graft tympanoplasty type 3. Laryngoscope 1965;75(09):1452-1461

10 Goodhill V. Tragal perichondrium and cartilage in tympanoplasty. Arch Otolaryngol 1967;85(05):480-491

11 Jansen C. CARTILAGE-TYMPANOPLASTY. Laryngoscope 1963; 73:1288-1301

12 Salen B. MYRINGOPLASTY USING SEPTUM CARTILAGE. Acta Otolaryngol Suppl 1964;188(SUPPL 188):188, 82

13 Adkins WY, Osguthorpe JD. Use of a composite autograft to prevent recurrent cholesteatoma caused by canal wall defects. Otolaryngol Head Neck Surg 1984;92(03):319-321

14 Chaudhary N, Anand N, Taperwal A, Rai AK. Role of autografts in the reconstruction of ossicular chain in intact canal wall procedures. Indian J Otolaryngol Head Neck Surg 2003;55(03):157-159

15 Yung M. Cartilage tympanoplasty: literature review. J Laryngol Otol 2008;122(07):663-672

16 Mohanty S, Gopinath M, Subramanian M, Vijayan N. Relevance of Pure Tone Average (PTA) as a Predictor for Incus Erosion. Indian J Otolaryngol Head Neck Surg 2012;64(04):374-376

17 Ebenezer J, Rupa V. Preoperative predictors of incudal necrosis in chronic suppurative otitis media. Otolaryngol Head Neck Surg 2010;142(03):415-420

18 Galy-Bernadoy C, Akkari M, Mathiolon C, Mondain M, Uziel A, Venail F. Comparison of early hearing outcomes of type 2 ossiculoplasty using hydroxyapatite bone cement versus other materials. Eur Ann Otorhinolaryngol Head Neck Dis 2014;131(05):289-292

19 Jayakumar CL, Inbaraj LR, Pinto GJO. Pre-operative Indicators of Ossicular Necrosis in Tubotympanic CSOM. Indian J Otolaryngol Head Neck Surg 2016;68(04):462-467 